\title{
Induction of Cancer By Viruses
}

\author{
by Renato Dulbecco
}

The transformation of the normal cells of an animal into cancer cells is a process which can be induced experimentally by a variety of agents. A perplexing aspect of these experiments is the difficulty, or impossibility, of formulating a unitary mechanism for the various agents. For instance, cancer can be induced equally well by substances which have a strong action on the nucleic acids, or by hormones, or even by completely inert substances, such as sheets of plastic inserted under the skin. The lack of similarity in the properties of cancer-inducing agents has suggested that the similarity lies in the cellular mechanisms that they affect.

Among the agents that induce cancer are viruses. We look at viruses as interesting objects for an experimental study of cancer because we understand much more about the interaction of cells with viruses than with any other cancerinducing agent. Furthermore, viruses are the only such agents that can induce cancers in dispersed cells cultivated in vitro. This result suggests that viruses induce cancer by direct action on the cells, whereas other carcinogens act indirectly and require the participation of mechanisms which operate at the level of the whole organism.

The nature of the changes caused by viruses in the transformation of normal cells into cancer cells can be well appreciated if one studies the properties of normal cells and of cancer cells in vitro. Cells are grown in dishes in contact with a solid substrate of glass or plastic, immersed in a liquid nutrient medium. Normal cells grow very rapidly as long as there is space between them; as soon as reciprocal contacts are established over a large part of the cell contour, growth stops. Therefore, these cells tend to constitute monolayers, cellular sheets which are one cell thick. Once the monolayer forms, growth stops.
This is not because the nutrients in the medium are exhausted, since the addition of more nutrients does not cause the growth to resume. Growth resumes if the cells are detached and transferred, more diluted, to a new dish, where they are not in reciprocal contact; or, simply, if part of the monolayer is mechanically removed. Therefore, cessation of growth is caused by regulatory mechanisms which are sensitive to the establishment of reciprocal contacts between cells.

The cellular surface appears to be the sensor: that detects the contact. This is suggested by cinematographic observations of cultures of normal cells, which show that the normal undulating movement of the margin of moving cells is immediately arrested when the margin touches the sirface of another cell. Similar mechanisms are probably responsible for maintaining the multiplication of the cells of the body under a strict control. The very existence of multicellular organisms depends on these regulatory mechanisms.

The behavior of cancer cells grown in vitro under the same conditions is totally different. These cells continue to grow after they have established a reciprocal contact and therefore are not confined to a monolayer. In effect, they form, in the dishes, very thick multilayered sheets, which stop growing when the food supply runs out or when toxic products of metabolism accumulate excessively. Regulation of cell multiplication is either absent or is ineffective in these cells. The absence of regulation of cancer cells is manifested, of course, in the organism, where these cells grow without any of the constraints which limit the multiplication of normal cells.

These characteristics of normal and of cancer cells are the basis for studying the effect of tumorinducing viruses in vitro. In fact, when, in a monolayer of normal cells, one of the cells is 
tain cellular genes. Thus, differentiation consists of the expression of the functionality of genes transformed into a cancer cell as a result of virus infection, it escapes the regulatory mechanisms and resumes growth. This cell gives rise to a colony of cells, a little cancer, which piles up on the background of the monolayer of normal cells. By taking advantage of this property, the tumorforming potency of a virus suspension can be determined.

These observations raise two interesting and interconnected questions: How does the virus cause the escape of the cells from regulatory influences? And what can we infer about these regulatory influences, of which we know very little, from the fact that they can be made ineffective in a virus-infected cell? There is a certain duality in these questions, since the same problem affects not only the understanding of the mode of action of tumor-producing viruses, but also the operation of the regulatory mechanisms. A similar duality often connects these two areas of biological research.

\section{Cancer viruses}

There are two main groups of cancer viruses, each one fairly homogeneous. The viruses of one group contain RNA as the genetic material, and those of the other group contain DNA. The properties of cancers produced by viruses of different groups are markedly different; those of cancers produced by viruses of the same group are quite similar, with one notable exception.

The pertinent findings can be summarized as follows. The RNA-containing viruses, except the Rous sarcoma virtis, produce differentiated tumors. The tumors are produced in a single step; that is, the cell which is infected becomes itself transformed into a cancer cell. The DNA-containing viruses produce undifferentiated tumors. The cancer cells are not those first infected by the virus, but derivatives of them which are formed after a number of cell generations.

\section{Differentiation}

During the development of an organism from a single cell - the fertilized egg - to the completely developed multicellular organism, cells appear which have more and more specialized functions. We call this process differentiation. The specialized functions are caused by the synthesis in the cells of specific molecules, essentially proteins, which are made on specification of cer-

which were previously inactive.

The sudden expression of a gene function is the consequence of the operation of regulatory mechanisms, which have been studied very extensively in bacteria. The results obtained in bacteria have shown that the expression of gene functions is prevented by specific intracellular repressors, which are in turn made by other genes; and that the expression of the genes can be resumed when the repressors cease to be made. Production of the repressor or its discontinuation is under the control of controller substances, of small molecular weight and of many kinds, which come from the outside.

\section{The pattern in animal cells}

Regulation of gene function in animal cells is believed to follow the same general pattern; the expression of genes is regulated by specific intracellular repressors. However, an important deviation from the bacterial case may exist in the mechanism by which the control of repression takes place. There is little evidence in animal cells for a controlling action of substances of small molecular weight and great variety coming from the outside.

The known substances controlling cellular properties in animal cells, presumably through a control of gene expression, belong to a few classes and are fairly large molecules. Among these substances we find hormones, some of which are steroids, others proteins; we find specific growth factors for certain cells of the nervous system and for the skin epithelium, which are fairly large proteins; we find the protein antigens which are able to elicit a specific antibody response.

It is not known at all whether these controlling substances are taken into the cells; it is quite possible that they act on the cell surface. In fact, the cell surface is a very active and selective system, which is responsible for introducing essentially everything the cell takes from the outside. Molecules and ions penetrate the cell through the action of specific permeases, which have the duplex function of recognizing the molecules, with an incredible amount of specificity, and of pumping them in against a concentration gradient. Modification of the function of specific permeases may very well have specific effects on the functionality of cellular genes, by modifying the internal concentration of certain small molecules coming from the outside. The regulatory action 
very early stage of differentiation give rise to of cell-to-cell contacts may also occur through similar mechanisms.

Another important aspect of differentiation is that the controlling substances can induce a certain type of differentiation only by acting on cells which have already reached another, well-cletermined, stage of differentiation. Thus, differentiation is a sequential process, which develops according to instructions that are coded in the genetic material of the cells. The various stages are presumably locked in by the operation of feedback mechanisms.

The ability of certain viruses to prodice differentiated cancers shows that these viruses alter the regulatory state of the cells without disrupting the complex instructions coded in the genetic material of the cells; thus, these viruses must act by causing a specific and functionally-well-circumscribed change of the cell. On the contrary, the production of undifferentiated cancers by other viruses can be attributed to more profound or less circumscribed disturbances.

\section{Effects of viruses on cells}

With these differences in mind we may now look a little more closely at the effects that the two types of viruses have on the cells they infect.

Let us look first at the RNA-containing viruses. Cells infected by viruses of this type, whether they produce tumors or not, synthesize a virusspecific protein which becomes incorporated in the cellular surface, as if it were a protein of the cells themselves. It is a very surprising observation that these cells, which contain a considerable proportion of viral protein in their surface, survive and in many cases multiply very actively.

In cancers produced by $\mathrm{RNA}$-containing viruses the viral RNA is present in all cells all the time; therefore, the synthesis of viral protein occurs constantly in these cells. The incorporation of the viral protein in the cellular surface may cause the alteration of specific functions; so, on this basis, the loss of response to factors regulating cellular multiplication can be understood, as well as the differentiation of the cells.

The differentiation of the cancer cells follows the pattern which is normal for the cells at the stage at which they were infected. For instance, precursors of white blood cells produce, after infection, tumors of white blood cells, which are called leukemias; precursors of bone-forming cells produce bone tumors; embryonic remnants at a of differentiation which normally occur. Thus, the virus acts as the controller substance responsible for actuating the type of differentiation of which the cell is capable; the differentiation attained is characteristic more of the type of cell affected than of the virus type.

A possible interpretation of these events is that the cell, owing to the alteration of its surface caused by the incorporation of viral protein, recognizes, as specific controllers of differentiation, substances which normally do not have that function. It is interesting that a similar point of view can be entertained to explain certain puzzling observations of experimental embryology.

\section{The Rous sarcoma virus}

The only RNA-containing virus which causes undifferentiated tumors is the Rous sarcoma virus. This virus also has an important peculiarity: it is defective, in the sense that its RNA, although capable of self-replication, is unable to give rise to the production of complete infectious virus particles. The nature of the defect is unknown, and it is not therefore possible to determine whether the presence of the defect is responsible for the lack of differentiation of the tumor produced by this virus. It is, however, conceivable that the defect consists of an alteration of the structure of the viral protein that becomes incorporated in the cellular surface; the altered protein might then cause unusually large changes in the properties of the cell surface, and thus destroy the fine balance required for maintaining a state of differentiation.

The DNA-containing viruses of small size, to which the tumor-producing viruses belong, are synthesized in the nucleus of the cell. They do not cause any extensive incorporation of viral material in the cellular surface, although some of them can cause other characteristic surface alterations. During the multiplication of the virus the cellular DNA suffers damages, especially by enzymes, which in some cases are found to he formed as a consequence of virus infection.

In the cancer cells induced by these viruses the viral DNA is not recognizable by our present means; it is, however, likely that some part of the viral DNA persists in these cells, because they have two characteristic properties which are absent in normal cells. One property is a special surface configuration, detectable immunologically; the other is the occurrence of breaks in the cellu- 
Cancer and Viruses . . . continued

lar DNA for many generations after infection, perhaps caused by the activity of a new enzyme. Both surface configuration and hypothetical enzyme may be the expression of information contained in the viral DNA which persists in the cells.

The transformation of cells by DNA-containing viruses takes place in at least two steps. The first step occurs immediately after infection: it affects the regulation of cell multiplication but not the state of differentiation, which remains unchanged. These first changes are probably similar to those induced by RNA-containing viruses, and may also be caused by modifications of the cell surface. The cells in which this first step has occurred, however, are not cancer cells. From them the true cancer cells emerge after a number of cell generations, during which many breaks take place in the cellinlar DNA. These cancer cells appear to carry permanent and profound alterations of their DNA, which can be detected in some cases by studying their chromosomes.

The formation of cancer cells by DNA-containing viruses is therefore a process involving the cell nucleus and causing structural alterations of the cellular DNA. It is likely that these alter- ations produce a disarrangement of the coordination between the function of the cellular genes, causing the lack of differentiation that characterizes the cancer cells produced by these viruses.

A conclusion that one can derive from these considerations is that probably there are two distinct types of cancer cells. In one type, exemplified by cells transformed by RNA-containing viruses, the cellular alteration is functional. If the viral RNA could be removed from the cells, they should revert to a state of normality; the cancer state is potentially reversible. In the other type, exemplified by cells transformed by DNAcontaining viruses, the cellular alteration is structural. Presumably the transformation would not revert even if the viral DNA were removed; the cancer state is irreversible.

These conclusions have stressed the significance of these investigations for the problem of the origin and nature of cancer. The point should, however, be made again that studies on the mechanism of cancer production by viruses have a much broader interest. In fact, they also offer experimental approaches for studying cellular regulation and differentiation, which are among the most outstanding insolved problems of biology today.



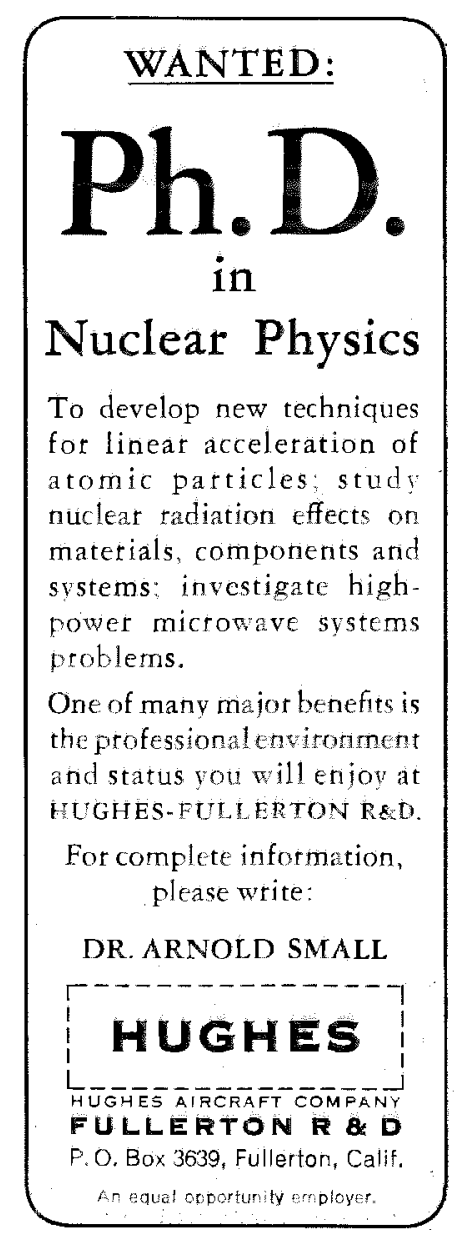

Engineering and Science 